\title{
Derivation of the near-surface dielectric function of amorphous silicon from photoelectron loss spectra
}

Denis David ${ }^{1}$, Christian Godet ${ }^{2}$, Hussein Sabbah $^{2}$, Soraya Ababou-Girard ${ }^{2}$, Francine Solal ${ }^{2}$, Virginia $\mathrm{Chu}^{3}$, João Pedro Conde ${ }^{3}$

${ }^{1}$ Instituto de Física, Universidade Federal da Bahia, Campus Universitário de Ondina, 40.210-340 Salvador, Bahia (Brazil)

${ }^{2}$ Physique des Surfaces et Interfaces, Institut de Physique de Rennes (CNRS UMR 6251), Université Rennes 1, Beaulieu - Bât. 11C - 35042 Rennes (France)

${ }^{3}$ INESC Microsistemas e Nanotechnologias and IN- Institute of Nanoscience and Nanotechnology, Rua Alves Redol, 9, 1000-029 Lisboa (Portugal)

e-mail: christian.godet@univ-rennes1.fr

PACS:

73.20.Mf Collective excitations: plasmons

73.60.Ht Photoemission: disordered structures

68.35.bj Surface structure: amorphous semiconductors, glasses

82.80.Pv Electron spectroscopy (XPS)

Keywords: XPS, plasmon, dielectric function, surface, amorphous silicon

Classifications: amorphous silicon, XPS 


\section{Abstract}

The near-surface dielectric function $\varepsilon(\hbar \omega)$ of hydrogenated amorphous silicon (a-Si:H) films has been derived from X-ray photoelectron energy-loss spectra, over the energy range 0-45 eV. Removal of low lying single-electron excitations is a prerequisite step to proceed to the derivation of the single plasmon energy loss function $\operatorname{Im}[-1 / \varepsilon(\hbar \omega)]$ due to collective electron oscillations. Several methods are compared to separate interband transitions from bulk or surface plasmons excitation. The shape of interband excitation loss in the range 1-10 eV can be described by a Henke function; alternatively, its removal using a sigmoid weighting function is a low-noise and reliable method. After deconvolution of multiple plasmon losses and self-consistent elimination of surface plasmon excitation, the single plasmon loss distribution allows recovery of optical (ellipsometry) data measured in the near-UV to visible range. 


\section{Introduction}

In semiconductor thin films and optical coatings, the dielectric function of the sub-surface region influences a variety of physical parameters (optical reflectivity, plasmon energy, dispersive component of the surface energy). Its determination is thus important for corresponding applications (plasmonics, antireflective or low emissivity coatings, adhesion).

This work is part of a global effort to derive the near-surface dielectric function, $\varepsilon(\hbar \omega)$, of amorphous semiconductors from Photo Electron Energy Loss Spectroscopy (PEELS). The energy-loss spectrum, observed after each elastic line in XPS spectra, results from inelastic collisions of ejected photoelectrons within the crossed material [1-6]. Careful removal of single-electron scattering (e.g. interband transitions) at low loss energy ( $\hbar \omega<10 \mathrm{eV}$ ) is a prerequisite step to derive the single plasmon loss distribution, $\operatorname{Im}[-1 / \varepsilon(\hbar \omega)]$, due to collective (plasmon) electron oscillations. Several methods are compared to separate interband transitions from bulk or surface plasmons excitation.

Since the bulk dielectric function of device-grade hydrogenated amorphous silicon (a$\mathrm{Si}: \mathrm{H})$ is reproducible and well known below $6 \mathrm{eV}$, this method is validated by comparing the dielectric functions $\varepsilon(\hbar \omega)$ of plasma-deposited a-Si:H obtained by PEELS (Si 2 p core level) and by spectroscopic ellipsometry (SE).

\section{Experimental methods}

Amorphous silicon films ( $\sim 50 \mathrm{~nm}$ thick) were grown at $250^{\circ} \mathrm{C}$ on $\mathrm{Si}(100)$ substrates using radiofrequency plasma decomposition of silane in a turbo-pumped high vacuum chamber (base pressure $\sim 10^{-6} \mathrm{~Pa}$ ). The a-Si:H film surface was protected against oxidation by spin coating of a $1 \mu \mathrm{m}$-thick photo-resist layer. Immediately before XPS measurements (or 
grafting process), it was dissolved in acetone and the surface silicon oxide was removed by immersion (90 seconds) in a buffered $40 \% \mathrm{NH}_{4} \mathrm{~F}$ solution out-gassed by argon bubbling.

SE data were obtained using a Horiba (UVISEL) spectrometer at $70^{\circ}$ incidence angle. XPS data were obtained with a VSW X-ray source $(\mathrm{Mg} \mathrm{K \alpha}, 1253.6 \mathrm{eV})$ and Omicron HA100 electron energy analyzer. By rotating the sample, angular analysis could be performed from $\alpha$ $=0^{\circ}$ (normal) to $65^{\circ}$ emission angles. Plasmon losses were measured with a $1.3 \mathrm{eV}$ resolution and a typical count rate of $8 \times 10^{4}$ counts $\mathrm{eV}^{-1}$, near the plasmon loss peak maximum [6-7]. The zero-loss peak, with kinetic energy $E_{0}$, is thus slightly broadened, with an apparent full width FWHM of $2.1 \mathrm{eV}$.

\section{Analysis of core level loss spectra}

A photoelectron loses energy because the induced electric field in the medium acts on the electron as it moves [1]. Collective excitations (plasmons) run as longitudinal charge density oscillations through the volume of the solid and along its surface. The energy distributions of bulk and surface plasmons are proportional to $\operatorname{Im}[-1 / \quad \varepsilon(\hbar \omega)]$ and to $\operatorname{Im}[(1 / \varepsilon(\hbar \omega))-$ $(4 / 1+\varepsilon(\hbar \omega))]$, where $\varepsilon(\hbar \omega)$ is the dielectric function; they are respectively peaked at the

plasma frequency $E_{\mathrm{P}}=\hbar \omega_{\mathrm{p}}$ and at $E_{\mathrm{sp}}=E_{\mathrm{P}} / \sqrt{2}$ [1]. Moreover, the intensity of the surface plasmon increases with emission angle $\alpha$, as $(1 / \cos \alpha)$.

This method to obtain $\varepsilon(\hbar \omega)$ is based on the dielectric formulation of the inelastic cross section for bulk and surface plasmons and the probability for bulk multiple plasmon losses. The practical method follows the technique developed by Egerton [1] for Electron Energy Loss Spectroscopy (EELS). However, correction factors $f(T)$ and $g(T)$ for bulk and surface loss distributions, specific to the XPS case (as opposed to EELS), are required to describe the 
anisotropy of photoelectron emission and scattering; this correction is important for the determination of the IMFP value [8]. The differential inelastic cross-section as a function of the photoelectron kinetic energy, $E=E_{0}-\hbar \omega=E_{0}-T$, is given by:

$$
\frac{\mathrm{d} \sigma}{\mathrm{d} E}=\frac{1}{2 \cdot \pi^{2} \cdot a_{0} \cdot E_{0} \cdot n_{A}} \cdot f(T) \cdot \operatorname{Im}\left(-\frac{1}{\varepsilon(\hbar \omega)}\right)
$$

with $f(T)=\pi \cdot\left(1-\frac{\sin ^{2} \gamma_{0}}{2}\right) \cdot \log \left(\frac{A(T)+1}{A(T)-1}\right)$ and $A(T)=\left(1-\frac{T}{2 \cdot E_{0}}\right) / \sqrt{1-\frac{T}{E_{0}}}$

$E_{0}$ being the initial kinetic energy of the non-relativistic photoelectron, $T$ the loss energy, $a_{0}$ the Bohr radius, and $n_{\mathrm{A}}$ the atom concentration.

For the recovery of the primary bulk loss spectrum from XPS data, a homogeneous depth distribution of electron emitters is assumed. Two major difficulties must be addressed: (i) an accurate elimination of the inelastic background arising from low energy single-electron scattering and multiple plasmon excitations at high loss energy $(\hbar \omega>20 \mathrm{eV})$; (ii) a clear identification of bulk and surface responses. In a previous work on $\mathrm{sp}^{3}$-rich amorphous carbon films, bulk and surface plasmon loss distributions, respectively $B(\hbar \omega)$ and $S(\hbar \omega)$, were derived from a simple parameterization of the measured angular loss spectra, $J(E)=B(\hbar \omega)+$ $S(\hbar \omega) / \cos \alpha$, without a priori assumptions on the shape of the distribution [6]. In addition, this study takes into account the relation between $B(\hbar \omega)$ and $S(\hbar \omega)$, and accurately eliminates single-electron interactions.

The inversion algorithm developed to determine the dielectric function from PEELS data is made of seven steps [8]: (i) in the case of non-monochromatic excitation, elimination of the contribution due to $\mathrm{Mg} \mathrm{K} \alpha$ source satellites; (ii) removal of the broad and strong core level elastic peak and the tail of single-electron losses, (iii) de-convolution of the multiple bulk plasmon losses using the Poisson distribution for multiple scattering given by Werner theory 
[4], (iv) normalization of the loss intensity using the optically measured value of $\operatorname{Re} \varepsilon(\hbar \omega=$ 0), (v) application of the Kramers-Krönig analysis in order to get the complex dielectric function $\varepsilon(\hbar \omega)$, and iterative separation of bulk and surface plasmon contributions until convergence is obtained, (vi) the inelastic mean free path (IMFP) value, $\lambda\left(E_{0}\right)$, is calculated using the correction factor $f(T)$, (vii) finally, Bethe sum rules are used to obtain the number of electrons per atom in the valence band, $N_{\mathrm{EFF}}$.

In this work, we focus on the most critical step (ii) by comparing four methods for the removal of single-electron scattering at low loss energies, typically in the range 1-10 eV.

In method (M1), a Gaussian or a Voigt function is fitted to the elastic peak profile and subtracted from the monochromatized experimental spectrum (circles in Fig. 1); if several chemical binding environments are present, a multiple-Gaussian adjustment can be made. In method (M2), the high-energy side of the elastic peak is subtracted from the low-energy side data, by a mirror operation, without fitting. Methods (M1) and (M2) reveal that, between the elastic peak and the plasmon peak, some signal remains with a typical width of $2 \mathrm{eV}$ (red curve in Fig. 1); it can be attributed to single-electron scattering at low loss energies, such as valence band electron excitation [9] or to an intrinsic loss mechanism [3].

In method (M3), the Henke model [9] for valence band electron excitation provides an analytic function, $y_{1}(T)$, at low energies; using the expansion $y_{2}(T) \approx a T$ of the plasmon distribution when $T$ tends to zero, the function

$$
\mathrm{y}(T)=\mathrm{y}_{1}(T)+\mathrm{y}_{2}(T)=\frac{\mathrm{d} T}{1+\mathrm{c} T^{\mathrm{m}}}+\mathrm{a} T
$$


can be fitted to the experimental signal in the range $0-10 \mathrm{eV}$, after subtracting the Gaussian function fitted to the zero loss peak. This separation procedure is rather accurate (Fig. 1); however, the plasmon signal in the $0-10 \mathrm{eV}$ range remains quite noisy (black dots in Fig. 2).

Method (M4) overcomes this difficulty by multiplying the experimental spectrum (including the zero-loss peak) by a sigmoid function (or error function) $\sigma(T)$ (red curve in Fig. 2):

$$
\sigma(T)=0 \text { for } T \leq E_{\mathrm{G}} \text { and } \sigma(T)=1-\exp \left(-\left(\frac{T-E_{G}}{\Delta E_{S I G}}\right)^{2}\right) \text { for } T>E_{\mathrm{G}}
$$

where $T$ is the loss energy and $E_{\mathrm{G}}$ the gap of the material that can be determined by optical methods. Note that the width of the adjusted function $y_{1}(T)$ can be used as a guess value for the sigmoid parameter $\Delta E_{\text {SIG. }}$

When the shape of the low-energy loss is unknown, using a sigmoid weighting function is a reliable method to remove the elastic peak and excitation of valence band electrons; it also suppresses the noise in the $0-10 \mathrm{eV}$ range while keeping the loss spectrum information (red plot in Fig. 2). The next sections show that this method is efficient for obtaining a dielectric function which recovers the independently measured optical data.

\section{Results}

The photoelectron energy-loss spectrum of a-Si:H at $\alpha=0^{\circ}$ is shown in Figure 3. The $\mathrm{Si}$ $2 \mathrm{p}$ spectrum at $\alpha=45^{\circ}$ shows no sign of surface oxidation. A bulk plasmon loss is clearly observed, with its maximum located at $17.2 \mathrm{eV}$ from the elastic peak (blue curve), and the second-order plasmon scattering is observed at $34.1 \mathrm{eV}$. Both values are consistent with previous reports $[\mathbf{1 0}, \mathbf{1 1}]$. The algorithm removes nearly completely the second-order plasmon 
peak. Note that there is no need for a specific baseline subtraction because the physical origin of the signal at high loss energies is now totally known.

The first order plasmon loss, $\operatorname{Im}[-1 / \varepsilon(\hbar \omega)]$ was calculated for data taken at various emission angles, using the index of refraction $n(\hbar \omega \sim 0)=3.40$. The algorithm was used either with or without taking into account the surface plasmon. When computing the surface plasmon, all $\operatorname{Im}[-1 / \varepsilon(\hbar \omega)]$ curves coincide better in the surface plasmon region from 5 to 15 $\mathrm{eV}$ (not shown). The surface plasmon of a-Si:H is located at $12.7 \mathrm{eV}$; in this sample it has a weak intensity which angular dependence can be fitted by a $(b / \cos \alpha)$ law, with $b=0.77$.

\section{Discussion}

A smooth cutoff has been applied to the low loss energy region of PEELS data by using either a Henke function or a sigmoid function; as shown in Figure 2, the remaining low energy loss signal has a linear dependence vs loss energy, expected for a plasmon loss near the band gap energy. Both Henke and sigmoid functions provide very similar electron energy loss functions. Fitting a Henke function is helpful to estimate $\Delta E_{\mathrm{SIG}}$ in the sigmoid function (Eqn. 3) which gives a better signal-to-noise ratio.

The comparison between $\varepsilon(\hbar \omega)$ deduced from PEELS and SE measurements on a-Si:H (Figure 4) is satisfactory. Although the dielectric function derived from PEELS is slightly narrower than SE optical data for clean a-Si:H obtained by Aspnes up to $6 \mathrm{eV}$ [12], it is close to our SE measurements at several energies on the same sample (squares in Figure 4). Confidence in the PEELS dielectric function is also supported by the Bethe sum rule which provides a number of valence electrons $N_{\mathrm{EFF}} \approx 4$. We estimate the accuracy on the position and the amplitude of maximum of $\varepsilon_{2}(E)$ as $E_{\max }=3.6 \pm 0.2 \mathrm{eV}$ and $\varepsilon_{2}\left(E_{\max }\right)=27 \pm 1.5$. 


\section{Conclusion}

Amorphous silicon (a-Si:H) has been used as a reference material to compare several methods for the separation of interband transitions and bulk or surface plasmons excitation. In PEELS, the accurate removal of low lying single electron excitations $(0-10 \mathrm{eV})$ is a prerequisite step in order to derive the single plasmon energy loss function $\operatorname{Im}[-1 / \varepsilon(\hbar \omega)]$ and the dielectric function $\varepsilon(\hbar \omega)$ over an energy range $0-45 \mathrm{eV}$. The shape of the valence band electron excitation loss is well described by a Henke function; removal of this quasi-elastic peak using a sigmoid weighting function is a low-noise and reliable method. The near-surface dielectric function of a-Si:H derived from PEELS data (depth sensitivity of $5 \mathrm{~nm}$ ) is close to the bulk optical data in the UV-visible range.

\section{Acknowledgments}

One of us (D.D.) is grateful to FAPESB (Bahia - Brazil) for funding part of this work. 


\section{References}

[1] R. F. Egerton, Electron Energy-Loss Spectroscopy in the Electron Microscope, Plenum Press (1996).

[2] S. Tougaard, Surf. Sci. 109 (1984) 208-218.

[3] A.C. Simonsen, F. Yubero, S. Tougaard, Phys. Rev. B 56 (1997) 1612-1619.

[4] W.S.M. Werner, Surf. Interface Anal. 31 (2001) 141-176.

[5] J. Schäfer, J. Ristein, R. Graupner, L. Ley, U. Stephan, T. Frauenheim, V.S. Veerasamy, G.A.J. Amaratunga, M. Weiler, H. Ehrardt, Phys. Rev. B 53 (1996) 7762.

[6] C. Godet, D. David, H. Sabbah, S. Ababou-Girard, F. Solal, Appl. Surf. Sci. 255 (2009) 6598-6606.

[7] H. Sabbah, J. P. Conde, V. Chu, A. Zebda, S. Ababou-Girard, F. Solal, C. Godet, J. Appl. Phys. 105 (2009) 064914.

[8] D. David, C. Godet, unpublished.

[9] B.L. Henke, J. Liesegang, S.D. Smith, Phys. Rev. B 19 (1979) 3004-3021.

[10] K.J. Grüntz, L. Ley, R.L. Johnson, Phys. Rev. B 24 (1981) 2069-2080.

[11] T. Bekkay, K. Piyakis, Y. Diawara, E. Sacher, A. Yelon, J.F. Currie, Surf. Sci. 258 (1991) 190-196.

[12] D.E. Aspnes, in Handbook of Optical constants of Solids II, edited by E.D. Palik (Academic, San Diego), 1985. 


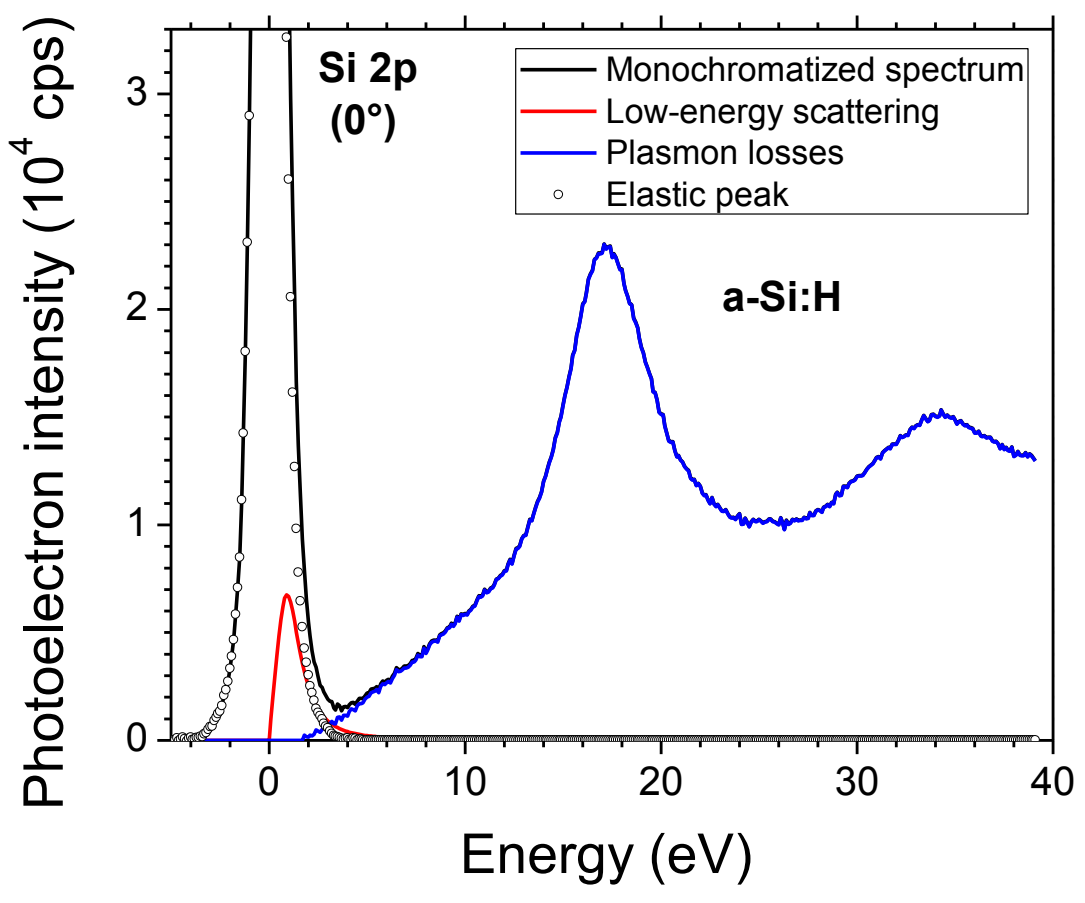

Fig. 1: Separation of the plasmon signal of a-Si:H by method M1: subtraction of a Gaussian function (circles) and method M3 : further subtraction of the low energy-loss signal by fitting $y(T)$ (Eqn. 2). 


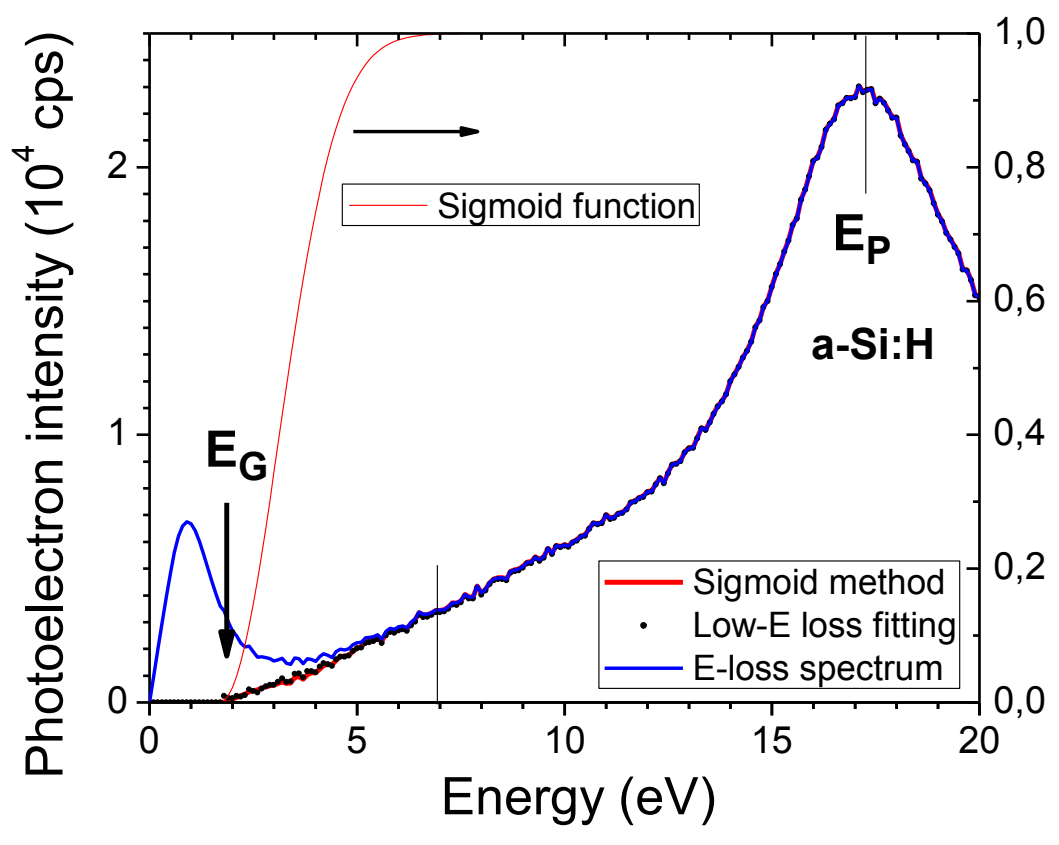

Fig. 2: Separation of the plasmon signal by methods M3 (fitting low-energy losses by a Henke function, Eqn. 2) and M4 (sigmoid method) with $\Delta E_{\mathrm{SIG}}=2.0 \mathrm{eV}, E_{\mathrm{G}}=1.7 \mathrm{eV}$. M3 and M4 results are overlapped; however method M4 completely suppresses the noise in the loss energy range 0-7 eV. 


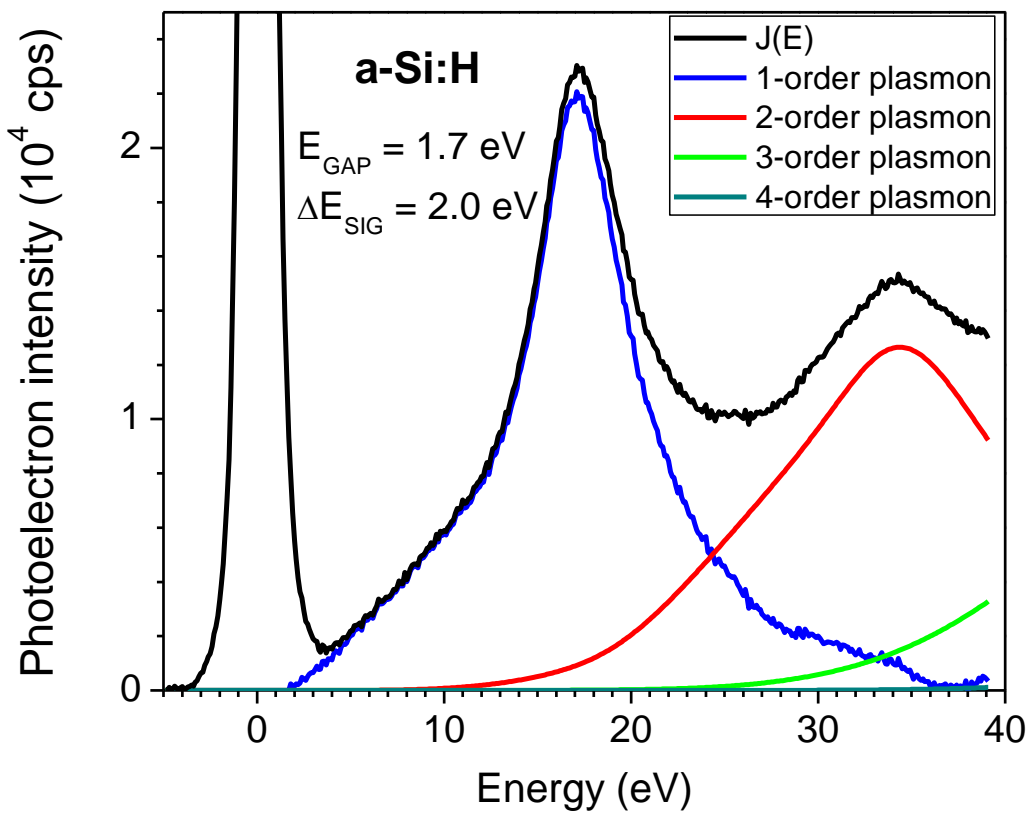

Fig. 3 : Decomposition (up to fourth-order) of the multiple-order plasmon loss obtained after application of method M4 to the Si 2p core level spectrum of a-Si:H. The single plasmon loss (blue curve) is nearly free of the second-order contribution at $34 \mathrm{eV}$. 

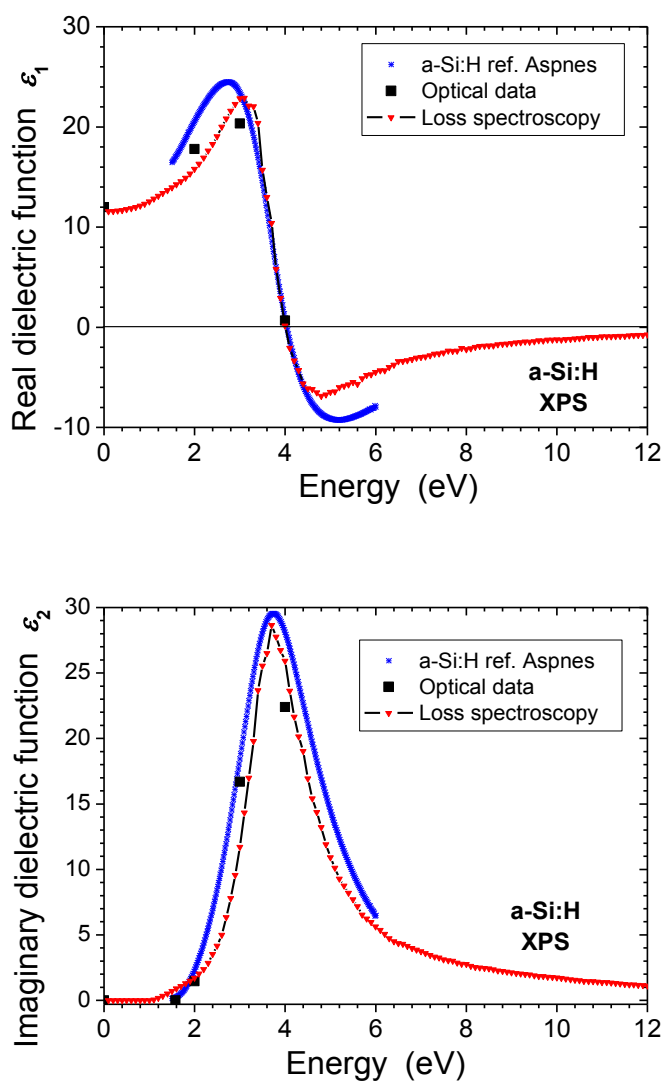

Fig. 4 : Dielectric function $\left(\varepsilon_{1}(\hbar \omega), \varepsilon_{2}(\hbar \omega)\right)$ derived from PEELS (red triangles) and spectroscopic ellipsometry (black squares: same sample; blue stars: a-Si:H from Aspnes [12] up to $6 \mathrm{eV})$. 